\title{
巴氯芬对慢性吗啡依赖后条件化位置偏好和戒断症状的抑制
}

\author{
牛海晨 ${ }^{1}$, 尚 涁 $^{2}$, 李 玲 $^{3}$, 孙宁䂞 ${ }^{1}$, 关一亮 ${ }^{4}$, 余化霖 ${ }^{2, *}$, 马原野 ${ }^{1}$ \\ (1. 中国科学院昆明动物研究所 灵长类认知实验室, 动物模型和人类疾病机理重点实验室, 云南 昆明 650223 ; \\ 2. 昆明医学院第一附属医院 神经外科, 云南 昆明 650118；3. 成都军区昆明总医院 医学影像科, 云南 昆明 650032; \\ 4. 内蒙古自治区公安厅禁毒总队, 呼和浩特 010051)
}

摘要：小鼠连续 7 天腹腔注射吗啡 $(40 \mathrm{mg} / \mathrm{kg})$ 建立条件化位置偏好模型，连续皮下递增注射吗啡（25、50、 75、100、125、150 mg/kg)，成瘾后腹腔注射纳络酮（6 mg/kg）诱导戒断症状（跳跃行为）建立戒断模型。腹腔 注射 $\mathrm{GABA}_{\mathrm{B}}$ 受体激动剂巴氯芬 $(2 \mathrm{mg} / \mathrm{kg}$ ) 可以有效地抑制吗啡诱导的条件化位置偏好和减轻纳络酮诱导的戒断 症状, 结果表明GABA系统参与动物成瘾后渴求和戒断过程, 激动 $\mathrm{GABA}_{\mathrm{B}}$ 受体可以在一定程度上抑制成瘾的心理 和生理戒断症状。

关键词: 吗啡; 成瘾; 戒断; 巴氯芬; 条件化位置偏好; GABA 受体; 纳络酮; 生理依赖; 心理依赖

中图分类号: Q189 文献标识码: A 文章编号: 0254-5853-(2008)06-0621-06

\section{Baclofen Inhibited the Morphine-induced Conditioned Placed Preference and Withdrawal Syndromes}

\author{
NIU Hai-chen ${ }^{1}$, SHANG Bin ${ }^{2}$, SUN Ning-lei ${ }^{1}$, LI Ling ${ }^{3}$, \\ GUAN Yi-liang ${ }^{4}$, YU Hua-lin ${ }^{2, *}$, MA Yuan-ye ${ }^{1}$ \\ (1. Laboratory of Primate Neuroscience Research and Key Laboratory of Animal Models and Human Disease Mechanisms, Kunming \\ Institute of Zoology, the Chinese Academy of Sciences, Kunming 650223, China; \\ 2. Neurosurgery office, the First Affiliated Hospital of Kunming Medical College, Kunming 650118, China; \\ 3. Medical Image Office, Kunming General Hospital of PLA, Kunming 650032, China; \\ 4. Narcotics Control Administration of Inner Mongolian Public Security Department, Huhhot 010051, China)
}

\begin{abstract}
The mice with a continuous 7-day intraperitoneal injection of morphine was induced the conditioned placed reference (CPP) model and the mice with a continuous 7-day subcutaneous injection of morphine $(25,50,75,100$, $125,150 \mathrm{mg} / \mathrm{kg}$ ) was induced withdrawal model (jump behavior) by naloxone administration $(6 \mathrm{mg} / \mathrm{kg}$ ). The results suggested that the administration of the $\mathrm{GABA}_{\mathrm{B}}$ receptor agonist, baclofen, could effectively attenuate the expression of $\mathrm{CPP}$ and the nalxone-induced response in the mice. It could be concluded that activating $\mathrm{GABA}_{\mathrm{B}}$ receptor can alleviate physical and psychic dependence syndromes to a certian extent .
\end{abstract}

Key word: Morphine; Addiction; Withdrawal; Baclofen; Conditioned placed preference; GABA receptor; Naloxone; Physiological dependence; Psychic-dependence

药物滥用是全球突出的社会和医学问题, 海洛 因、吗啡、杜冷丁等阿片类物质是主要滥用药物, 长期药物滥用引起吸食者药物依赖和神经系统损 害(Hyman \& Malenka, 2001), 给社会和家庭带来严 重的不良后果。
毒品吸食患者对阿片类药物的依赖性，包括心 理依赖性和生理依赖性, 主要表现为高复吸率和停 药后的受药行为。心理依赖性表现为不同程度的心 理渴求以及强迫用药行为(Narita et al, 2005)。人类 的心理依赖性表现为复吸和渴求行为, 可以由多种 
因素引发, 如药物和药物相关的环境线索 (Carter \& Tiffany, 1999)。实验动物的依赖性可用条件化位置 偏好模型 (conditioned place preference, CPP)、自身 给药行为模型 (self-administration, SA)、药物辨别 (drug discrimination, DD) 等行为学模型进行检测 (Barkov, 1986)。生理依赖性主要表现为不同程度的 戒断症状, 吸食者可表现出许多不适的身体戒断症 状。实验动物可用量化的戒断体征进行量化评分, 如大鼠可用湿狗样颤抖、齿颤、眼睑下垂、咀嚼、 直立、扭体、躯体拉伸等行为指标检测, 小鼠可用 纳络酮催发跳跃症状(Sinchaisuk \& Rockhold, 2002) 检测。

目前认为阿片类药物成瘾是一个涉及多脑区 多神经递质的过程 (阿片系统、多巴胺能系统、谷 氨酸能系统、 $\operatorname{GABA}(\gamma$ 氨基丁酸)系统和去甲肾上腺 素系统等), 大量的研究实验表明脑内药物奖赏或 强化的主要结构为中脑一边缘多巴胺系统(See et al, 2007; Margolis et al, 2006)该系统是阿片类药物发挥 兴奋性作用的轴心, 是强化效应的最后通路。大量 事实表明, 激活伏隔核 (NAc)、前额叶 (PFC)、 腹侧背盖区 (VTA)、杏仁核、腹侧海马区的多巴 胺和谷氨酸通路, 在线索环境或药物诱导的阿片类 药物复吸中发挥重要作用(Kalivas \& McFarland, 2003)。然而近期行为学研究表明激活GABA可以有 效地抑制奖赏行为, 例如非选择性GABA激动剂 Vigabatrin在可卡因诱发的自身给药行为中, 通过降 低奖赏作用发挥的抑制自身给药行为形成的作用 (Filip et al, 2007), 进一步的实验表明通过巴氯芬直 接激活背侧额叶、腹侧苍白球(McFarland \& Kalivas, 2001)、腹侧海马区和腹侧VTA区(Rogers \& See, 2007)的 $\mathrm{GABA}_{\mathrm{B}}$ 受体具有抑制环境诱发的复吸行 为。 $\mathrm{GABA}_{\mathrm{B}}$ 受体激动剂可以通过抑制VTA区谷氨酸 能神经元和多巴胺神经元的递质释放而改变阿片 类药物的兴奋性和诱导复吸的特性(Alden et al, 2002)。动物长期使用阿片类物质能够诱导阿片敏感 细胞发生cAMP依赖性的级联反应, 该反应是急性 戒断期间的行为学反应基础 (vidor-Reiss et al, 1997)。以前报道认为巴氯芬能够干扰海马区、中脑 区和VTA区的多巴胺代谢(Santiago et al, 1993), 通 过增加脑内多巴胺含量缓解停药后的戒断行为。

Walker \& Koob (2007) 研究发现巴氯芬可以降低酒 精依赖停药后的戒断症状, 但在阿片类药物成瘾后 戒断症状研究至今仍少见。基于上述研究, 本研究
分两部分: 以小鼠腹腔注射巴氯芬为给药手段, 以 CPP模型为检测模型, 研究巴氯芬对吗啡成瘾后心 理成瘾的影响; 利用小鼠腹腔注射巴氯芬, 探讨对 吗啡成瘾后纳络酮诱发戒断症状的影响。本研究拟 为药物治疗生理戒断提供实验基础

\section{1 材料和方法}

\section{1 实验动物}

ICR 小鼠, 雄性, $18-22 \mathrm{~g}$, SPF（无特殊病原 体) 级, 购自昆明医学院动物科, 许可证 $\operatorname{SCXK}$ (滇) 2002-0013, 饲养在动物笼内, 自由进水、进食, 自然光照, 室温 $(25 \pm 2){ }^{\circ} \mathrm{C}$, 操作符合动物管理条 例。实验动物共分为 4 组: 生理盐水组、吗啡组、 $1 \mathrm{mg} / \mathrm{kg}$ 巴氯芬十吗啡组和 $2 \mathrm{mg} / \mathrm{kg}$ 巴氯芬十吗啡 组, 每组 8 只。

\section{2 实验药品}

盐酸吗啡注射液 (沈阳第一制药厂, 产品批号: 040305), 巴氯芬（化学名称：4-氨基-3-4-氯苯 基 -丁酸, 浙江天衡药业有限公司, 国药准字 H:19980104), 盐酸纳络酮注射液（北京四环制药 有限公司, 国药准字 H43021669)。

\section{3 实验装置 (CPP 系统)}

CPP 系统由位置偏好行为测试箱和视频记录检 测系统组成。行为测试箱由 2 个大小相同的试验箱 $(30 \mathrm{~cm} \times 30 \mathrm{~cm} \times 35 \mathrm{~cm})$ 和中间过渡箱组成 $(30 \mathrm{~cm} \times 10$ $\mathrm{cm} \times 35 \mathrm{~cm})$ 。一个试验箱四周为黑白相间的纵向条 形图案 (宽 $3 \mathrm{~cm}$, 长 $34 \mathrm{~cm}$ ), 箱底质地光滑; 另一 个试验箱四周为同宽度黑白相间的横向条形图案， 箱底质地粗粘; 过渡箱形成两个试验箱之间的通 道, 在与试验箱连接处有可上下滑动的挡片 (10 $\mathrm{cm} \times 34.5 \mathrm{~cm}$ ), 可以把任一试验箱和过渡箱隔离开。 过渡箱墙壁为灰色。视频记录检测系统包括安置于 测试箱上方的红外监控摄像头和计算机软件系统, 小鼠行为数据由计算机软件记录并自动分析。

\section{4 实验操作程序}

\subsection{1 巴氯芬对吗啡诱导的条件性位置偏好的抑 制作用}

a. 条件化前测试: 将 ICR 小鼠逐只放入 CPP 箱中间过渡箱内, 抽出两侧活动隔板让动物在两侧 试验箱和过渡箱内自由探索 $15 \mathrm{~min}$, 每天 1 次, 连 续 3 天; 第 3 天计算机记录动物在每侧试验箱中停 留时间, 淘汰对任一试验箱具有明显偏好的动物 (任一试验箱中停留时间 $>12 \mathrm{~min}$ 或 $<3 \mathrm{~min}$ )。将 箱底质地光滑的试验箱定为阳性箱, 质地粗粘的定 义为阴性箱, 把篮选后的动物按照在阳性箱内停留 
时间随机分组。

b. 条件化训练: 用隔板把两个试验箱和过渡箱 隔开, 药物组动物腹腔注射盐酸吗啡 $40 \mathrm{mg} / \mathrm{kg}$ 后放 入阳性箱内适应 $30 \mathrm{~min}$, 取出放回饲养笼; $6 \mathrm{~h}$ 后腹 腔注射同体积生理盐水, 放入阴性箱中适应 $30 \mathrm{~min}$, 生理盐水组阴性箱和阳性箱内训练均腹腔注射 0.1 $\mathrm{mL}$ 生理盐水, 连续训练 7 天。

c. 条件化测试: 第 8 天取下测试箱与过渡箱之 间的隔板进行位置偏好测试, 测试前 $15 \mathrm{~min}$ 吗啡组 和生理盐水组腹腔注射 $0.1 \mathrm{~mL}$ 生理盐水, 巴氯芬组 按照分组剂量腹腔注射巴氯芬 $1.0 \mathrm{mg} / \mathrm{kg}$ 和 2.0 $\mathrm{mg} / \mathrm{kg}$ 。然后将小鼠放入过渡箱内允许动物在测试 箱内自由活动 $15 \mathrm{~min}$ 并由计算机记录动物在阳性箱 中停留时间。

1.4.2巴氯芬对吗啡依赖后纳络酮诱导身体戒断 症状影响 ICR 雄性小鼠(体重 18-22 g), 随机分 组, 每组 8 只。分别为生理盐水组、吗啡戒断组、 $1 \mathrm{mg} / \mathrm{kg}$ 巴氯芬十吗啡组、 $2 \mathrm{mg} / \mathrm{kg}$ 巴氯芬十吗啡组。 生理盐水组动物每次皮下注射生理盐水 $0.1 \mathrm{~mL}$ 后 放入饲养笼中, 每天 3 次, 给药时间分别为 8:00、 13:00 和 18:00, 连续给药时间为 6 天, 第 7 天早晨 皮下注射 $0.1 \mathrm{~mL}$ 生理盐水后 $4 \mathrm{~h}$ 腹腔注射 $6 \mathrm{mg} / \mathrm{kg}$ 纳络酮, 记录 $5 \mathrm{~min}$ 跳跃次数。吗啡的注射方法为 每组小鼠连续 6 天皮下注射吗啡, 每天注射吗啡剂 量分别为 $25 、 50 、 75 、 100 、 125$ 和 $150 \mathrm{mg} / \mathrm{kg}$, 注 射时间同生理盐水组, 注射吗啡后将小鼠放入饲养 笼中, 第 7 天早晨皮下注射吗啡剂量维持 150 $\mathrm{mg} / \mathrm{kg}$ 。4h 后吗啡戒断组腹腔注射 $0.1 \mathrm{~mL}$ 生理盐水, 其余各组腹腔注射巴氯芬, 剂量为 $1.0 \mathrm{mg} / \mathrm{kg}$ 和 2.0 $\mathrm{mg} / \mathrm{kg}$, 各组 $15 \mathrm{~min}$ 后腹腔注射 $6 \mathrm{mg} / \mathrm{kg}$ 纳络酮, 然 后立刻记录 $5 \mathrm{~min}$ 跳跃次数。

\section{5 数据分析}

$\mathrm{CPP}$ 箱阳性箱停留时间以秒 ( $\mathrm{s}$ ) 为记录单位, 采用 SPSS 13.0 为统计工具, 对阳性箱停留时间进 行多元方差的重复分析。戒断症状跳跃以次为记录 单位, 戒断症状观察指标由 SPSS 单因素方差做组 间分析, $P<0.05$ 为有统计学差异。

\section{2 结 果}

\section{1 巴氯芬对吗啡诱导的条件性位置偏好的抑制 作用}

各组之间小鼠条件化前测试期在阳性箱的停 留时间无差异, 表明各组小鼠在进行药物处理前无
差异, 接受吗啡注射的小鼠在阳性箱停留时间长于 生理盐水组, 表明CPP模型建立成功 $\left(F_{(3,27)}=3.122\right.$, $P<0.05$ )。事后药物组比较检验表明, $1 \mathrm{mg} / \mathrm{kg}$ 巴氯 芬对吗啡诱导的CPP无抑制作用 $(P>0.05)$, 而 2 $\mathrm{mg} / \mathrm{kg}$ 巴氯芬对吗啡诱导的 CPP 具有抑制作用 $(P<0.05)$ 。当不考虑随着时间延长 $\mathrm{CPP}$ 形成的自然 消退影响, $1 \mathrm{mg} / \mathrm{kg}$ 巴氯芬组与吗啡组比较在第 3 天 表现出抑制作用 $(P<0.05), 2 \mathrm{mg} / \mathrm{kg}$ 巴氯芬组与吗 啡组比较显示在第 1 天接近显著 $(P=0.077)$, 第 3 天表现出抑制作用 $(P<0.05)$ 。药物剂量和时间双因 素分析表明 $2 \mathrm{mg} / \mathrm{kg}$ 巴氯芬组对CPP的形成具有一 定的抑制作用(图 1)。

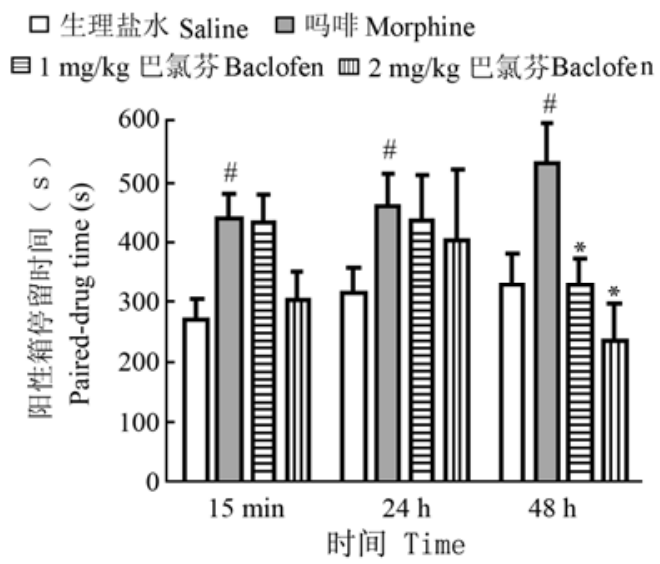

图 1 腹腔注射不同剂量巴氯芬 $15 \mathrm{~min} 、 24$ 和 $48 \mathrm{~h}$ 后, 对吗啡诱导产生条件化位置偏好的小鼠在阳性箱 停留时间的影响

Fig. 1 Baclofen inhibited the expression of morphineinduced CPP in mice

\#表示吗啡+盐水组与生理盐水组比较, 差异显著(one-way ANOVA);

*表示事后药物组与吗啡组比较差异显著(LSD)。

\# Means there was a significant difference between morphine + saline group and saline group (one-way ANOVA) and * means there was a significant difference in the post hoc test (LSD).

\section{2 巴氯芬对吗啡依赖后纳络酮诱导身体戒断症 状影响}

小鼠递增剂量皮下注射吗啡在纳络酮作用下 诱发跳跃行为, 如图 2 所示。小鼠连续注射吗啡后 在纳络酮诱导下跳跃次数明显增加 $\left[F_{(3,34)}=6.072\right.$, $P<0.05]$, 事后比较检验显示吗啡戒断组与生理盐水 组差异极显著 $(P<0.001)$, 纳络酩诱导小鼠停药后 戒断模型成立。同时吗啡组与 $2 \mathrm{mg} / \mathrm{kg}$ 巴氯芬组差异 显著 $(P<0.05), 2 \mathrm{mg} / \mathrm{kg}$ 巴氯芬表现出对纳络酮诱 导的吗啡戒断后跳跃症状的抑制作用, 然而 $1 \mathrm{mg} / \mathrm{kg}$ 巴氯芬却无此抑制作用 $(P>0.05)$, 巴氯芬对纳络酮 
诱导的吗啡戒断后的抑制症状存在一定的剂量依 赖关系。

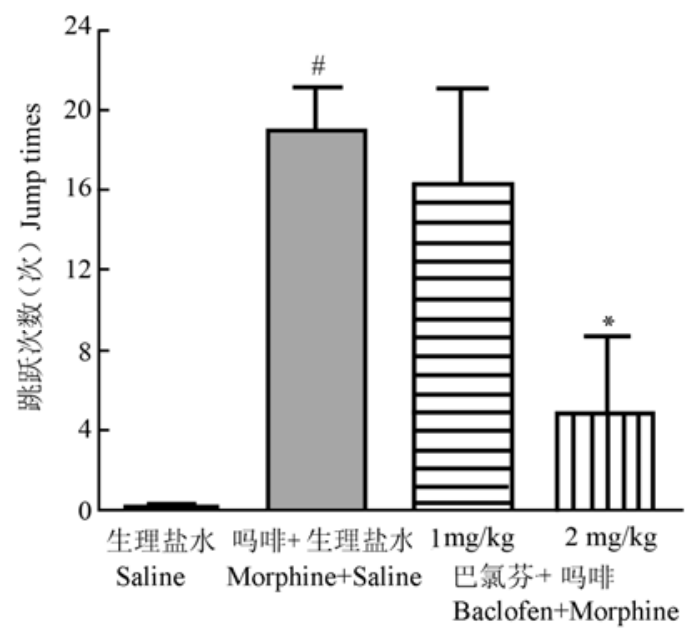

图 2 巴氯芬减少吗啡戒断过程中的跳跃行为

Fig. 2 Baclofen attenuate the naloxone-induced response \#表示吗啡+盐水组与生理盐水组比较差异显著(one-way ANOVA); *表 示事后药物组与吗啡组比较差异显著(LSD)。

\# means there was a significant difference between morphine + saline group and saline group (one-way ANOVA) and * means there was a significant difference in the post hoc test(LSD).

\section{3 讨 论}

条件性位置偏好模型是评价受药行为或心理 渴求的经典动物模型, 用来判定某种物质的奖赏效 应和精神依赖潜力。它是通过训练使动物建立起药 物的奖赏效应与某一特定环境刺激之间的一种条 件性联系, 训练后动物会偏好停留在曾经给予药物 奖赏的环境中。其理论基础为: 情感性刺激如奖赏 或惩罚可以诱导机体产生趋向或回避行为反应, 如 果该情感性刺激与中性环境刺激结合, 则可以使得 中性环境刺激得与情感刺激相似的能力, 即也能诱 导机体产生趋向或避开刺激的行为反应(McBride et al, 1999)。因此, 将药物作用与某种环境条件结合 以后, 则可以通过动物对该环境条件的行为反应来 判断该药物的奖赏或惩罚效应。本实验腹腔注射巴 氯芬后的小鼠在阳性箱内的停留时间较吗啡组减 少, 则可以推断巴氯芬具有减弱吗啡诱导的心理渴 求的作用。

以前研究中, 慢性吗啡处理较多采用的是皮下 埋植、皮下注射、腹腔注射等方式, 剂量恒定或渐 增, 隔日、每日注射一次或每日两次注射。本研究 中, 慢性吗啡处理方式参考了多篇文献, 并结合本
实验室多年的慢性吗啡动物实验研究结果确定, 从 药物使用方式、次数、剂量等尽量模拟阿片依赖者 的滥用模式, 以期用动物实验的结果进一步说明临 床相关问题。本研究采用非平衡 CPP 程序, 能更好 地展示动物训练后对偏好侧的转变, 也使 $\mathrm{CPP}$ 实验 较为容易进行。本研究用 $40 \mathrm{mg} / \mathrm{kg}$ 恒定剂量的吗啡 腹腔注射小鼠使之与两隔箱的不同特征间产生条 件反射, 很快就使小鼠对阳性箱产生了偏好, 表现 为吗啡组小鼠在伴药侧停留的时间明显长于对照 组, 表明吗啡诱导动物产生了偏好。Zarrindast et al (2006) 报道海马 CA1 区局部注射巴氯芬既不能建 立 CPP, 也不能产生条件化位置厌恶 (CPA), 但能 够降低吗啡诱导的 CPP 形成, 这与我们腹腔注射巴 氯芬抑制 CPP 形成的结果一致。吗啡通过解除 GABA 对 DA 神经元的抑制作用而发挥提高 DA 释 放量的作用, 从而产生吗啡的奖赏效应; 巴氯芬增 强 GABA 神经抑制性从而到达减弱 CPP 形成的效 果(Brebner et al, 2002)。同时本实验中小鼠连续递增 剂量皮下注射吗啡, 停药后在 $6 \mathrm{mg} / \mathrm{kg}$ 纳络酮作用 下可以诱导跳跃的戒断症状, 结果与 Diaz et al (2003)实验一致, 表明巴氯芬也可以抑制吗啡停药 后的生理依赖行为。

脑内GABA受体主要集中在脑灰质区, 其受体 分为 $\mathrm{GABA}_{\mathrm{A}}$ 受体, $\mathrm{GABA}_{\mathrm{B}}$ 受体及 $\mathrm{GABA}_{\mathrm{C}}$ 受体三类。 有研究显示 $\mathrm{GABA}$ 参与了阿片类药物的奖赏效应, 在 伏隔核一腹侧苍白球 (nucleus accumbens-pallidum, NAc-VP) 通路中神经递质是 $\mathrm{GABA}$, 而腹侧苍白球的功能就是表达NAc兴奋时 的行为活动, 损毁大鼠双侧苍白球后, 自身给予海 洛因的行为明显减少 (Spano et al, 2007)。阿片系统 通过解除VTA区的GABA对DA神经元的抑制作用 而发挥增加 DA释放的作用 (Anden \& Wachtel, 1977)。我们的结果表明腹腔注射巴氯芬能够抑制吗 啡诱导 CPP的形成, 同时有文献报道巴氯芬不能产 生条件化位置厌恶的反应(Diaz et al,2003), 这些结 果与以前巴氯芬抑制吗啡诱导的压杆反应相一致 (Tsuji et al,1996)。

除此之外, 研究认为吗啡成瘾过程与学习记忆 过程密切相关( Lu et al, 2000), 海马和中脑组成的功 能环路激活能够增强长时程增强 (LTP) 和学习过 程, 海马CA1 区注射巴氯芬能够损害学习过程, 减 低吗啡诱导CPP形成。在CPP模型中, $\mathrm{GABA}_{\mathrm{B}}$ 受体 拮抗剂CGP 35348 and phaclofen能够增强大鼠的学 
习能力, 增强大鼠脑片的LTP形成(Mondadori et al, 1993)。因此认为 $\mathrm{GABA}_{\mathrm{B}}$ 受体激动剂巴氯芬可能产 生与其拮抗剂相反的作用, 减弱或损坏大鼠脑片的 LTP形成, 降低动物的学习能力, 从而使环境相关 线索不能将奖赏相关记忆提取出来的作用, 结果导 致CPP形成受到抑制。对于巴氯芬抑制CPP形成机 制的另一种解释, 巴氯芬可能通过GABA或谷氨酸 抑制中枢系统 $\mathrm{Ca}^{2+}$ 、激活 $\mathrm{K}^{+}$等途径, 影响学习记忆 级联反映的传导 (Kalivas,1993), 研究已经证实 $\mathrm{GABA}_{\mathrm{B}}$ 受体激动剂的应答机制为 $\mathrm{GABA}$ 与 $\mathrm{GABA}_{\mathrm{B}}$ 受体结合后通过 $\mathrm{GABA}_{\mathrm{B}}$ 受体一 $\mathrm{G}$ 蛋白藕联的 $\mathrm{K}^{+}$、 $\mathrm{Ca}^{2+}$ 通道以及腺苷酸环化酶 $(\mathrm{CA})$ 而产生应答效应。 应答效应表现为 $\mathrm{K}^{+}$转导增加, 而 $\mathrm{Ca}^{2+}$ 转导抑制 (Pfeiffer \& Zhang, 2007)。

阿片肽是一类肽类物质, 由神经细胞释放后扩 散到周围靶细胞与其受体结合, 通过膜受体信号转 导系统, 转而改变膜的兴奋性或其他递质的释放产 生相应的生理效应。在阿片依赖中起作用的膜受体 信号转导系统主要是膜受体-cAMP-蛋白激酶系 统(Nestler, 1992)。以 $\mathrm{Ca}^{2+}$ 为第二信使的信号转导系 统也发挥一定的作用并且与 $\mathrm{GABA}_{\mathrm{B}}$ 受体的相互作 用关系尤为密切。通常情况下, 阿片与受体结合后 对cAMP信号转导系统起抑制作用, 而阿片药物戒 断时, 膜受体一 CAMP一蛋白激酶信号转导系统的 活性水平上调从而使整个神经元出现依赖和戒断 的生物效应, 这就是 G蛋白一 $\mathrm{CAMP}$ 一蛋白激酶系统 在阿片类依赖和戒断中的基本过程(Han et al, 2006)。长期给予阿片类物质并形成依赖后, 阿片类

\section{参考文献:}

Alden TD, Lytle RA, Park TS, Noetzel MJ, Ojemann JG. 2002. Intrathecal baclofen withdrawal: A case report and review of the literature [J]. Childs Nerv Syst, 18: 522-525.

Anden NE, Wachtel H. 1977. Biochemical effects of baclofen (beta-parachlorophenyl-GABA) on the dopamine and the noradrenaline in the rat brain[J]. Acta Pharmacol Toxicol (Copenh), 40: 310-320.

Brebner K, Rose AC, Roberts DC. 2002. A potential role for GABA(B) agonists in the treatment of psychostimulant addiction[J]. Alcohol, 37(5): 478-484.

Brendan MWalker, George FKoob. 2007. The $\gamma$-aminobutyric acid-B receptor agonist baclofen attenuates responding for ethanol in ethanol-dependent rats[J]. Alcohol Clin Exp Res, 31(1): 11-18.

Carter BL, Tiffany ST. 1999. Meta-analysis of cue-reactivity in addiction research [J]. Addiction, 94: 327-340.

Diaz SL, Kemmling AK, Balerio GN. 2003. Baclofen reestablishes striatal and cortical dopamine concentrations during naloxone-precipitated withdrawal [J]. Neurochem Int, 42: 293-298.
物质使该系统发生适应, 逐渐上调形成稳态, 这时 突然中断阿片类的给予, 即阿片类戒断, 上调的 $\mathrm{G}$ 蛋白一cAMP一蛋白激酶系统失去阿片类的抑制而 导致稳态失稳, 该系统即发生急剧增高, 从而出现 一系列的戒断现象(Han et al, 2006), 临床上可以观 察到明显的戒断症状, 总称为戒断综合症, 研究表 明 $\mathrm{GABA}_{\mathrm{B}}$ 受体与腺苷酸环化酶（CA）相互作用后 能够限制CA活性而使突触后抑制延长便抑制了上 调的cAMP系统，从而降低了阿片的戒断症状(Helm et al, 2005)。很多研究表明在动物戒断期间许多神 经递质如谷氨酸、去甲肾上腺素释放增加(Simonato, 1996; Done et al, 1992), 而同时GABA的释放量减少 (Simonato, 1996)。GABA释放量的减少可以部分地 增加胞外腺苷的含量, 腺苷通过cAMP通路进一步 增加递质的释放而增强戒断症状。巴氯芬可能通过 增加GABA的释放量进而抑制递质的释放达到减缓 戒断症状的作用。在本研究中生理盐水组注射 6 $\mathrm{mg} / \mathrm{kg}$ 纳络酮并不能诱发小鼠跳跃症状, 表明纳络 酮本身对其行为没有影响, 对于吗啡依赖动物应用 纳络酮后能够诱发跳跃症状原因可能是依赖动物 内源性阿片系统机制参与的原因。

本研究表明, 腹腔注射巴氯芬, 抑制了小鼠吗 啡诱导的条件性位置偏好, 也减少了小鼠在吗啡戒 断过程中的跳跃行为。虽然巴氯芬抑制CPP形成和 戒断症状的准确机制还需要进一步探讨, 但是本研 究说明, $\mathrm{GABA}_{\mathrm{B}}$ 受体有望成为药物治疗阿片类成瘾 心理渴求和生理渴求的作用靶点。

Done C, Silverstone P, Sharp T.1992. Effect of naloxone-precipitated morphine withdrawal on noradrenaline

release in rat hippocampus in vivo[J]. European Journal of Pharmacology, 215: 333-336.

Filip M, Frankowska M, Zaniewska M, Golda A, Przegalinski E, Vetulani J. 2007. Diverse effects of GABA-mimetic drugs on cocaine-evoked self-administration and discriminative stimulus effects in rats $[\mathrm{J}]$. Psychopharmacology (Berl), 192: 17-26.

Han MH, Bolanos CA, Green TA, Olson VG, Neve RL, Liu RJ, Aghajanian GK, Nestler EJ. 2006. Role of cAMP response element-binding protein in the rat locus ceruleus: Regulation of neuronal activity and opiate withdrawal behaviors[J]. J Neurosci, 26: 4624-4629.

Helm KA, Haberman RP, Dean SL, Hoyt EC, Melcher T, Lund PK, Gallagher M. 2005. GABAB receptor antagonist SGS742 improves spatial memory and reduces protein binding to the cAMP response element (CRE) in the hippocampus[J]. Neuropharmacology, 48: 956-964. 
Hyman SE, Malenka RC. 2001. Addiction and the brain: The neurobiology of compulsion and its persistence[J]. Nat Rev Neurosci, 2: 695-703.

Kalivas PW, McFarland K. 2003. Brain circuitry and the reinstatement of cocaine-seeking behavior [J]. Psychopharmacology (Berl) 168: 44-56.

Margolis EB, Lock H, Chefer VI, Shippenberg TS, Hjelmstad GO, Fields HL. 2006. $\kappa$ Opioids selectively control dopaminergic neurons projecting to the prefrontal cortex [J]. Proc Natl Acad Sci USA, 103(8): 2938-2942.

McBride WJ, Murphy JM, Ikemoto S. 1999. Localization of brain reinforcement mechanisms: Intracranial self-administration and intracranial place-conditioning studies [J]. Behav Brain Res, 101: 129-152.

McFarland K, Kalivas PW. 2001. The circuitry mediating cocaine-induced reinstatement of drug-seeking behavior [J]. J Neurosci, 21: 8655-8663.

Mondadori C, Jaekel J, Preiswerk G. 1993. CGP 36742: the first orally active GABAB blocker improves the cognitive performance of mice, rats, and rhesus monkeys [J]. Behav Neural Biol, 60: 62-68.

Narita M, Kato H, Miyoshi K, Aoki T, Yajima Y, Suzuki T. 2005. Treatment for psychological dependence on morphine: Usefulness of inhibiting NMDA receptor and its associated protein kinase in the nucleus accumbens [J]. Life Sci, 77: 2207-2220.

Nestler EJ. 1992. Molecular mechanisms of drug addiction[J]. J Neurosci, 12: $2439-2450$.

Pfeiffer A, Zhang W. 2007. Postnatal development of GABAB-receptor-mediated modulation of potassium currents in brainstem respiratory network of mouse[J]. Respir Physiol Neurobiol, 158: $22-29$.

Rogers JL, See RE. 2007. Selective inactivation of the ventral hippocampus attenuates cue-induced and cocaine-primed reinstatement of drug-seeking in rats [J]. Neurobiol Learn Mem, 87: 688-692.

Santiago M, Machado A, Cano J. 1993. In vivo release of dopamine from rat striatum, substantia nigra and prefrontal cortex: Differential modulation by baclofen [J]. Br J Pharmacol, 109: 814-818.

See RE, Elliott JC, Feltenstein MW. 2007. The role of dorsal vs ventral striatal pathways in cocaine-seeking behavior after prolonged abstinence in rats[J]. Psychopharmacology, 194(3): 321-331.

Simonato M. 1996. The neurochemistry of morphine addiction in the neocortex[J]. Trends in Pharmacological Sciences, 17: 410-415.

Sinchaisuk, Rockhold RW. 2002. Focal kappa-opioid receptor-mediated dependence and withdrawal in the nucleus paragigantocellularis [J]. Pharmacol Biochem Behav, 74: 241-252.

Spano MS, Fattore L, Fratta W, Fadda P. 2007. The GABAB receptor agonist baclofen prevents heroin-induced reinstatement of heroin-seeking behavior in rats[J]. Neuropharmacology, 52: 1555-1562.

Tsuji M, Nakagawa Y, Ishibashi Y, Yoshii T, Takashima T, Shimada M, Suzuki T. 1996. Activation of ventral tegmental GABAB receptors inhibits morphine-induced place preference in rats[J]. Eur J Pharmacol, 313: 169-173.

vidor-Reiss T, Nevo I, Saya D, Bayewitch M, Vogel Z. 1997. Opiate-induced adenylyl cyclase superactivation is isozyme-specific [J]. J Biol Chem, 272: 5040-5047.

Zarrindast MR, Massoudi R, Sepehri H, Rezayof A. 2006. Involvement of $\mathrm{GABA}_{\mathrm{B}}$ receptors of the dorsal hippocampus on the acquisition and expression of morphine-induced place preference in rats[J]. Physiol Behav, 87(1): 31-38. 